\title{
Long-term surveillance biopsy: Is it necessary after pediatric heart transplant?
}

\author{
David M. Peng ${ }^{1,2}$ (D) | Victoria Y. Ding ${ }^{3}$ | Seth A. Hollander ${ }^{1,2}$ | Tigran Khalapyan ${ }^{4}$ | \\ John C. Dykes ${ }^{1,2}$ | David N. Rosenthal ${ }^{1,2}$ | Christopher S. Almond ${ }^{1,2,4}$ | \\ Charlotte Sakarovitch $^{3}$ | Manisha Desai ${ }^{3}$ | Doff B. McElhinney ${ }^{1,2,4,5}$
}

\author{
${ }^{1}$ Department of Pediatrics, Stanford \\ University School of Medicine, Palo Alto, \\ California \\ ${ }^{2}$ Betty Irene Moore Children's Heart Center, \\ Palo Alto, California \\ ${ }^{3}$ Department of Medicine, Stanford \\ University School of Medicine, Palo Alto, \\ California \\ ${ }^{4}$ Clinical and Translational Research \\ Program, Palo Alto, California \\ ${ }^{5}$ Department of Cardiothoracic \\ Surgery, Stanford University School of \\ Medicine, Palo Alto, California \\ Correspondence \\ David M. Peng, CS Mott Children's Hospital, \\ Ann Arbor, MI. \\ Email: davepeng@med.umich.edu
}

\section{Present Address}

David M. Peng, Division of Pediatric

Cardiology, Department of

Pediatrics, University of Michigan, Ann

Arbor, Michigan

\begin{abstract}
Due to limited and conflicting data in pediatric patients, long-term routine surveillance endomyocardial biopsy (RSB) in pediatric heart transplant (HT) remains controversial. We sought to characterize the rate of positive RSB and determine factors associated with RSB-detected rejection. Records of patients transplanted at a single institution from 1995 to 2015 with >2 year of post-HT biopsy data were reviewed for RSB-detected rejections occurring $>2$ year post- $\mathrm{HT}$. We illustrated the trajectory of significant rejections (ISHLT Grade $\geq 3 \mathrm{~A} / 2 \mathrm{R}$ ) among total RSB performed over time and used multivariable logistic regression to model the association between time and risk of rejection. We estimated Kaplan-Meier freedom from rejection rates by patient characteristics and used the log-rank test to assess differences in rejection probabilities. We identified the best-fitting Cox proportional hazards regression model. In 140 patients, 86\% did not have any episodes of significant RSB-detected rejection $>2$ year post- $\mathrm{HT}$. The overall empirical rate of RSB-detected rejection >2 year post-HT was 2.9/100 patient-years. The percentage of rejection among 815 RSB was $2.6 \%$ and remained stable over time. Years since transplant remained unassociated with rejection risk after adjusting for patient characteristics $(\mathrm{OR}=0.98 ; 95 \% \mathrm{Cl} 0.78-1.23 ; P=0.86)$. Older age at HT was the only factor that remained significantly associated with risk of RSB-detected rejection under multivariable Cox analysis $(P=0.008)$. Most pediatric patients did not have RSBdetected rejection beyond 2 years post-HT, and the majority of those who did were older at time of HT. Indiscriminate long-term RSB in pediatric heart transplant should be reconsidered given the low rate of detected rejection.

KEYWORDS

biopsy, heart transplantation, pediatrics, rejection
\end{abstract}

\section{1 | BACKGROUND}

First described by Dr. Caves in $1973,{ }^{1}$ the percutaneous, transvenous endomyocardial biopsy remains the gold standard for monitoring

Abbreviations: AMR, antibody-mediated rejection; $\mathrm{CHD}$, congenital heart disease; $\mathrm{Cl}$, confidence interval; HR, hazard ratio; HT, heart transplant; ISHLT, International Society for Heart and Lung Transplantation; OR, odds ratio; PHTS, Pediatric Heart Transplant Society; RSB, routine surveillance biopsy. allograft rejection after $\mathrm{HT}^{2}$ There are still, however, many disadvantages of this test including high cost, ${ }^{3}$ patient discomfort and inconvenience, rare risk of serious complication, ${ }^{4}$ and subjective interpretation of findings. ${ }^{5}$

Both adult and pediatric heart transplant recipients are at the highest risk for rejection early after $\mathrm{HT}^{6-8}$ Based on the rare risk of late rejection (mostly ranging between $1.6 \%$ and $3.7 \%$ ), most adult studies 
have supported discontinuing RSB 1-2 years after transplant in standard risk patients. ${ }^{3,6,9-23}$ In pediatric studies, late rejection is an important finding as it has been associated with decreased graft survival. ${ }^{24,25}$ The rate of rejection found by RSB in the second year and beyond post- $\mathrm{HT}$ is significantly more variable, ranging anywhere from $0 \%$ to 12\%. ${ }^{7,8,24-35}$ These previous studies (from before 2000) may not reflect current practices and risk and generally did not assess the possible time-related risk of late rejection. Due to the conflicting historical data and the absence of contemporary studies in pediatric heart recipients, the practice of long-term RSB in pediatric HT remains controversial.

The primary aims of this study were (a) to characterize the observed rate of positive routine surveillance endomyocardial biopsy in order to assess long-term surveillance protocol performance (beyond 2 years post-HT) and (b) to determine risk factors associated with surveillance-detected rejection.

\section{2 | METHODS}

\section{1 | Study population}

The inception cohort for this study included HT patients from a pediatric heart transplant center with demographic and post-transplant biopsy history available from institutional electronic health records from January 1995 through July 2015. In order to assess the relationship of early rejection to later rejection risk, we excluded patients with no recorded biopsy results within their first 60 days of transplant. Given our specific aims, we excluded patients who did not have at least 2 full years of post-HT biopsy data. We also excluded retransplant recipients. Further details regarding transplant outcomes in this cohort have been previously reported. ${ }^{36}$ The study was approved by the Institutional Review Board.

\section{2 | Induction and maintenance immunosuppression}

Standard protocol consisted of induction with methylprednisolone intraoperatively and either daclizumab, basiliximab, or rabbit antithymoglobulin. Postoperatively, patients received maintenance immunosuppressive therapy consisting of a calcineurin inhibitor (cyclosporine or tacrolimus), mycophenolate mofetil, and methylprednisolone or prednisone, which was tapered over the first post-transplant year. Cyclosporine and tacrolimus dosing were modulated to achieve target troughs of $300-350$ or $10-12 \mathrm{~g} / \mathrm{dL}$, respectively, for the first three post-transplant months, after which doses were adjusted downward sequentially to maintenance goal troughs of $175-225 \mathrm{~g} / \mathrm{dL}$ for cyclosporine or 5-7 g/dL for tacrolimus at $>24$ months post-transplant. Select patients were transitioned to sirolimus at various time points at least 6 months post-transplant and were dosed to achieve a target trough of $6-8 \mathrm{~g} / \mathrm{dL}$ and continued on reduced-dose cyclosporine or tacrolimus adjusted to achieve target troughs of $100-150$ or 2-4 g/dL, respectively.

\section{3 | Outcome}

Biopsies were classified according to the ISHLT 1990 criteria, $^{37}$ a standardized grading system to diagnose acute cellular rejection developed by the ISHLT. We considered grades 0 and $1 \mathrm{~A}$ as "negative for rejection" and $1 \mathrm{~B}$ and 2 as "mild rejection." Grades 3A, 3B, and 4 (equivalent to $2 R-3 R$ rejection using the revised 2004 grading scheme ${ }^{38}$ ) were classified as "clinically significant rejection."

Per institutional protocol, after 2 years post-transplant, surveillance biopsies have been routinely performed every 6 months during the study period irrespective of age. However, biopsies were often performed more frequently than every 6 months in patients perceived to be at higher risk for rejection or require heightened surveillance. For example, earlier or more frequent biopsies were performed in patients to follow up a rejection episode, with history of recurrent rejection, with other clinical concern for rejection, after significant changes in immunosuppression, with non-adherence and at the discretion of the team. It was not always clearly defined whether the more frequent biopsies were performed for regular, heightened surveillance, to follow up a rejection episode, or for active concern for new rejection. Thus, all biopsies performed earlier than the protocol guidelines were considered "non-routine." In order to focus the analysis on only routine, standard risk biopsies and assess the performance of an ongoing biannual RSB schedule, only biopsies occurring at least 4.5 months (135 days) after the previous biopsy were considered RSB.

\section{4 | Statistical analysis}

We presented baseline demographics at the time of HT, as well as early rejection history (years 0-2 post-HT), by highest achieved 1990 ISHLT grade category during follow-up (beyond Year 2). Categorical variables were summarized as counts and percentages, and continuous variables were summarized as median with first and third quartiles (Q1,3). Age at transplant and transplant era were categorized based on previously used thresholds. ${ }^{39}$

To characterize the rate of positive RSB in our time trend analysis, we first calculated and graphed the percentage of clinically significant rejections among total RSB performed from Year 2 to Year 10 post-transplant, overall and also stratified by age-group at HT. We then fit a multivariable logistic regression model to relate the log odds of identifying ISHLT grade of 3A or above to time since Year 2 of transplant, while accounting for transplant era, age at transplant, gender, CHD diagnosis, and history of rejection, as captured by baseline rejection status and cumulative number of biopsies at ISHLT grade $3 \mathrm{~A}$ or above during follow-up. Fractional polynomial regression, implemented via the $\mathrm{mfp} \mathrm{R}$ package (version 1.5.2, $\mathrm{R}$ Foundation for Statistical Computing, Vienna, Austria), was used to select the best functional form for modeling time. We estimated Huber-White standard errors to account for within-subject correlation and used them to construct $95 \% \mathrm{Cls}$ for estimated ORs.

To determine risk factors associated with surveillance-detected rejection, we estimated Kaplan-Meier freedom from rejection rates 
by patient characteristics and used the log-rank test to assess differences in survival probabilities. We then used exhaustive search model selection, implemented via the glmulti R package (version 1.0.7), to identify the Cox proportional hazards regression model among all possible models that minimized the Akaike information criterion and presented estimated $\mathrm{HRs}$ and associated $95 \% \mathrm{Cls}$ from this model. In these analyses, we considered as the outcome time to each patient's first occurrence of grade $\geq 3 \mathrm{~A}$ rejection during followup, censored at each patient's date of last biopsy or death. Statistical significance was assessed at the 0.05 level, and all analyses were performed in the R statistical computing framework (version 3.3). ${ }^{26}$

\section{3 | RESULTS}

Of 230 pediatric patients with both demographic and post-HT biopsy history available in the electronic medical record, 10 who did not have recorded biopsy results within the first 60 days post-HT and 80 others who did not have at least 2 years of post-HT follow-up were excluded ( 35 patients were transplanted within 2 years of the analysis, 25 patients died within 2 years post-HT, and another 20 did not have a full 2 years of follow-up for other reasons). This yielded an analytic cohort of 140 patients (Figure 1). Baseline demographics for the analytic cohort are shown in Table 1. Approximately half of the patients were male, and $38 \%$ were of white race. The median age at transplant was 10.6 (Q1,3: 2.3, 14.6) years. Approximately one-third of the patients had CHD and the majority (64\%) were transplanted in 2004 or later.

The 140 study patients underwent a total of 1916 biopsies beyond 2 years post-HT, of which 815 (43\%) were RSB. The median number of follow-up RSB per patient was 5 (Q1,3: 3, 9). Patients were followed for a total of 714.5 years, with median duration of follow-up 4.6 (Q1, 3: $2.6,7.5)$ years. Most patients $(86 \%)$ did not have any episodes of clinically significant rejection ( $\geq 3$ A by 1990 ISHLT grade) detected by RSB beyond 2 years post-HT. Characteristics of patients who did and did not have late rejection on RSB are summarized in Table 1.

The overall empirical rate of rejection detected by RSB after 2 years was 2.9 per 100 patient-years. The percentage of rejections among RSB was $2.6 \%$ and remained relatively stable over time (Figure 2A). Follow-up rejection patterns differed by age at HT (Figure 2B). In all children aged 2 or less at HT, there was a single rejection episode, which occurred in the third year post-HT. Four total rejection episodes were detected in the cohort between ages 2 and 13 at $\mathrm{HT}$. In patients over age 13 at $\mathrm{HT}$, the annual rate of rejection by RSB was higher, ranging from 0 to $15 \%$ over time.

After fitting a Cox proportional hazards model with adjustment for transplant era, age at transplant, gender, CHD diagnosis, and history of rejection, time since $\mathrm{HT}$ remained unassociated with rejection by RSB (OR $=0.98 ; 95 \% \mathrm{Cl} 0.78-1.23 ; P=0.86$, Table 2). The same model showed that patients over age 13 years at HT were significantly more likely to have rejection diagnosed by RSB compared with patients aged 2 years or less $(P=0.03)$.
FIGURE 1 CONSORT diagram of pediatric heart transplant study population

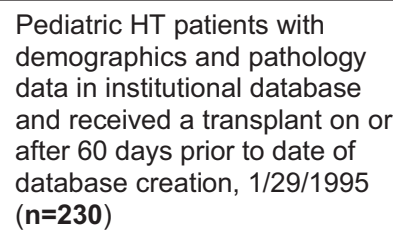

*Biopsies performed after two years were considered non-routine if done within 135 days of the previous biopsy. 


\begin{tabular}{|c|c|c|c|}
\hline & Overall ( $N=140)$ & $\begin{array}{l}\text { None or mild } \\
\text { rejection }{ }^{\mathrm{a}}(\mathrm{N}=121)\end{array}$ & $\begin{array}{l}\text { Clinically significant } \\
\text { rejection }^{\text {b }}(\mathrm{N}=19)\end{array}$ \\
\hline & $n(\%)$ & $\mathrm{n}(\%)$ & $\mathrm{n}(\%)$ \\
\hline \multicolumn{4}{|l|}{ Demographics } \\
\hline Male & $77(55.0)$ & $65(53.7)$ & $12(63.2)$ \\
\hline \multicolumn{4}{|l|}{ Patient race } \\
\hline White & $53(37.9)$ & $46(38.0)$ & $7(36.8)$ \\
\hline Black & $9(6.4)$ & $8(6.6)$ & $1(5.3)$ \\
\hline Other ${ }^{c}$ & $34(24.3)$ & $30(24.8)$ & $4(21.1)$ \\
\hline Unknown & $44(31.4)$ & $37(30.6)$ & $7(36.8)$ \\
\hline $\begin{array}{l}\text { Age at transplant, } \\
\text { years (median, } \\
\text { Q1,3) }\end{array}$ & $10.6(2.3,14.6)$ & $9.8(2.1,14.1)$ & $14.8(11.6,15.7)$ \\
\hline \multicolumn{4}{|c|}{ Age group at transplant } \\
\hline $0-2$ & $31(22.1)$ & $30(24.8)$ & $1(5.3)$ \\
\hline$>2-13$ & $58(41.4)$ & $52(43.0)$ & $6(31.6)$ \\
\hline$>13-23$ & $51(36.4)$ & $39(32.2)$ & $12(63.2)$ \\
\hline \multicolumn{4}{|l|}{ Transplant era } \\
\hline $1994-2003$ & $51(36.4)$ & $39(32.2)$ & $12(63.2)$ \\
\hline $2004-2008$ & $50(35.7)$ & $47(38.8)$ & $3(15.8)$ \\
\hline 2009-2013 & $39(27.9)$ & 35 (28.9) & $4(21.1)$ \\
\hline CHD diagnosis & $46(32.9)$ & $44(36.4)$ & 2 (10.5) \\
\hline \multicolumn{4}{|c|}{ Highest baseline ${ }^{d}$ rejection grade } \\
\hline Grade 1A & $75(53.6)$ & $66(54.5)$ & $9(47.4)$ \\
\hline Grade 1B & $3(2.1)$ & $2(1.7)$ & $1(5.3)$ \\
\hline Grade 2 & $19(13.6)$ & $15(12.4)$ & $4(21.1)$ \\
\hline Grade $\geq 3 A$ & $37(26.4)$ & $32(26.4)$ & $5(26.3)$ \\
\hline
\end{tabular}

TAB LE 1 Baseline characteristics of $\mathrm{N}=140$ patients by highest rejection grade identified beyond 2 y post- $\mathrm{HT}$

a1990 Grade $\leq 2$.

b1990 Grade $\geq 3$ A.

'Other race includes Asian, Latino, and Native American.

${ }^{d}$ Within first 2 y post-heart transplant.

Kaplan-Meier curves depicting time to first grade $\geq 3 \mathrm{~A}$ rejection by levels of patient characteristics are shown in Figure 3. Log-rank analysis identified age category at $\mathrm{HT}$ and $\mathrm{CHD}$ diagnosis as factors univariately associated with time to first occurrence of clinically significant rejection by RSB. Exhaustive search over all patient characteristics in Table 1 yielded the same two factors, and age category at HT was the only one that remained significantly associated with risk of rejection under multivariable Cox analysis ( $P=0.008$, Table 3$)$.

\section{4 | DISCUSSION}

This study demonstrates that most pediatric patients transplanted at our center between 1995 and 2015 did not have significant rejection detected by RSB beyond 2 years after $\mathrm{HT}$. The overall rate of rejection detected by RSB after 2 years was 2.9 per 100 patient-years. Older age at HT was the only factor associated with increased rejection detected by RSB on multivariable analysis. The rate of rejection by RSB did not change across transplant eras or decrease over time after transplant (within 10 years) in the study cohort.

A recent PHTS analysis showed that RSB detected $81.6 \%$ of the reported rejection episodes. ${ }^{26}$ However, since the PHTS does not collect the number of biopsies performed, the report could not provide the yield of RSB or address how frequently RSB should be performed. This updated pediatric study, which includes data and practices from the earliest era to the current, is the first to specifically investigate the utility and yield of a typical long-term surveillance biopsy protocol and identify factors associated with cellular rejection detected by RSB. Additionally, this time-related analysis is unique in that it utilized fractional polynomial methodology to determine the most appropriate functional form for modeling time, which allowed us to avoid assuming any particular trajectory, linear or otherwise for rejection over time.

There have not been any contemporary pediatric studies of RSB performance using data after 2000. Consistent with earlier studies, our analysis confirms that RSB is exceedingly low yield in patients 


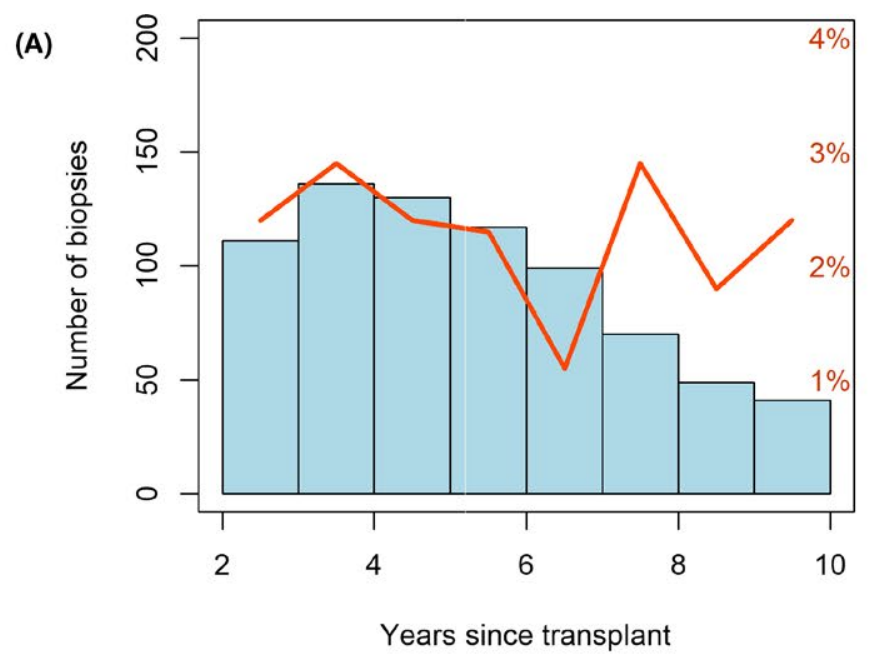

(B)

Age 0-2 Years

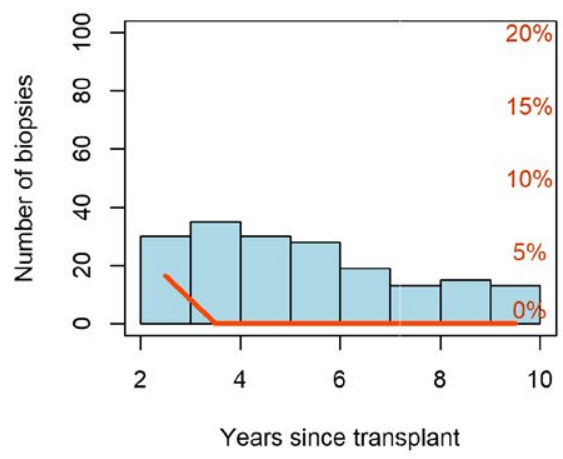

Age $>13-23$ Years

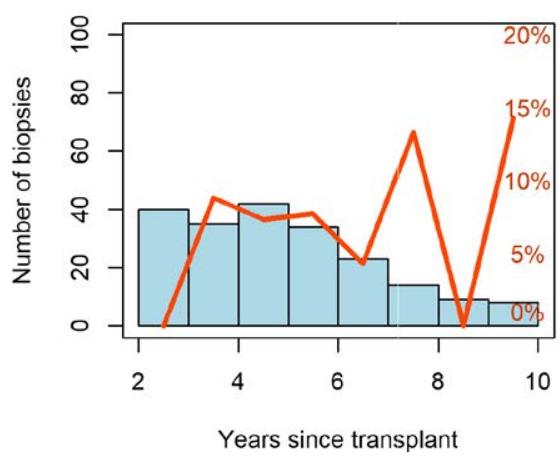

Age $>2-13$ Years

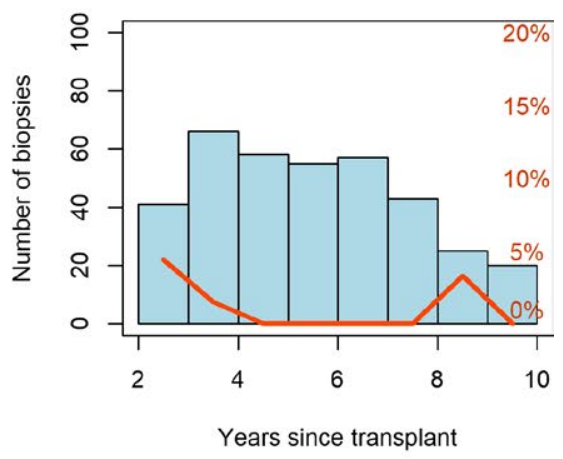

FIGURE 2 A, Total number of post-transplant routine biopsies (histogram) and percent of grade $\geq 3$ A routine biopsies (line) during followup, truncated at Year 10 due to subsequent low counts. B, Total number of post-transplant routine biopsies (histogram) and percent of grade $\geq 3 \mathrm{~A}$ routine biopsies (line) during follow-up, truncated at Year 10 due to subsequent low counts, stratified by transplant age categories

transplanted as infants. ${ }^{32,33}$ There was only a single episode of rejection by RSB detected in patients $\leq 2$ years old at the time of HT, occurring during the third year post-HT. In children transplanted between 2 and 13 years of age, the rate of rejection detected by RSB was also exceedingly low. On the other hand, late rejection detected by RSB was higher in patients $>13$ years old at HT. This finding is consistent with previous PHTS studies that have demonstrated older age to be a risk factor for both late rejection and rejection with severe hemodynamic compromise. ${ }^{8,24}$ In older patients, we hypothesize that differences in immunology, non-adherence and psychosocial stressors, donor-related factors, and other unknown influences may potentially contribute to the higher persistent risk of rejection, even late post- $\mathrm{HT}$.

In combination with prior reports, the current study shows that long-term (beyond 2 years post-HT) RSB for asymptomatic acute cellular rejection is low yield and suggests that RSB is not clearly indicated in younger children, especially those aged 2 years or less at HT. In older patients, our data suggest that 
TAB LE 2 Estimated ORs with robust 95\% Cls from multivariable logistic regression model to characterize surveillance performance over time

\begin{tabular}{|c|c|c|c|}
\hline Covariates & OR & $95 \% \mathrm{Cl}$ & $P$-value \\
\hline Years since baseline & 0.98 & $(0.78,1.23)$ & 0.861 \\
\hline \multicolumn{4}{|l|}{ Transplant era } \\
\hline $1995-2003$ & 1.00 & REF & REF \\
\hline $2004-2008$ & 0.68 & $(0.12,3.83)$ & 0.665 \\
\hline $2009-2013$ & 1.53 & $(0.36,6.44)$ & 0.559 \\
\hline \multicolumn{4}{|c|}{ Age-group at transplant (y) } \\
\hline $0-2$ & 1.00 & REF & REF \\
\hline$>2-13$ & 3.25 & $(0.39,27.1)$ & 0.277 \\
\hline$>13-23$ & 9.87 & $(1.19,81.6)$ & 0.034 \\
\hline Male & 0.79 & $(0.32,1.94)$ & 0.604 \\
\hline CHD diagnosis & 0.46 & $(0.08,2.53)$ & 0.370 \\
\hline $\begin{array}{l}\text { Cumulative no. of } \\
\geq 3 \mathrm{~A} \text { rejections }\end{array}$ & 1.63 & $(0.86,3.09)$ & 0.132 \\
\hline \multicolumn{4}{|c|}{ Baseline rejection category } \\
\hline $\begin{array}{l}\text { No rejection }(0, \\
1 \mathrm{~A})\end{array}$ & 1.00 & REF & REF \\
\hline $\begin{array}{l}\text { Mild rejection } \\
\text { only }(1 \mathrm{~B}, 2)\end{array}$ & 1.22 & $(0.35,4.29)$ & 0.754 \\
\hline $\begin{array}{l}\text { Grade } \geq 3 \mathrm{~A} \\
\text { rejection }\end{array}$ & 0.74 & $(0.12,4.60)$ & 0.750 \\
\hline
\end{tabular}

ongoing surveillance may be warranted. Previous studies have shown that earlier era, previous rejection, and non-white race were associated with a higher risk of rejection. ${ }^{24,25}$ These factors were not associated with risk for rejection in this single-center analysis, which focused only on RSB and intentionally omitted patients at higher perceived risk who received more frequent biopsies. Rejection episodes detected by clinically indicated biopsies (performed more frequently than standard protocol) were not included in the analysis. Not unexpectedly, the number of early rejection episodes (within 2 years post-HT) in our cohort is lower than what has been previously described. ${ }^{8}$ If early rejecters were placed back on standard surveillance protocol, presumably due to clinical stability, they were not at higher risk for a positive RSB in our data.

Unexpectedly, there was a trend toward lower rate of rejection in patients with CHD. This borderline association most likely reflects the fact that the CHD patients were younger at the time of HT (median age 7.5 years vs 11.4 years) and younger age at HT is associated with decreased rejection. The association of $\mathrm{CHD}$ with rejection risk was no longer significant in multivariable analysis that included age.

In this analysis, more than half of the biopsies were obtained more frequently than required by the protocol. At our center, patients often received additional biopsies for immunosuppression changes, rejection follow-up, history of recurrent rejection, noncompliance, and, of course, clinical concern for rejection. We intentionally excluded these biopsies to specifically analyze the routine, standard risk biopsies.

\section{1 | Limitations}

There are limitations to this study. The data were from a single center. However, current multicenter registry data do not capture the granularity necessary to answer the questions posed by our study. Relatedly, because the number of patients and events are relatively small in this single-center analysis, we cannot draw definitive conclusions and make large-scale practice changes based on the findings. The analysis also excluded 20 patients who did have complete 2 years of follow-up for unclear reasons which may have introduced bias into the findings. In addition, there may have been some misclassification of biopsies as routine (ie, a biopsy performed $\geq 4.5$ months after the previous biopsy may have been "non-routine" or clinically indicated). However, we believe this was the most consistent and straightforward way to classify biopsies, as the documentation was not always explicit and our institutional protocol called for a biopsy to be performed biannually after 2 years post-HT. This classification scheme also enabled us to analyze the performance of a commonly performed biopsy protocol. Since clinically indicated, non-RSB are expected to have higher detection rates, misclassification of non-RSB as RSB would be expected to produce an overestimate of the rate of detection with RSB. The true rejection rates with RSB may be even lower than described. We acknowledge that these data may not include some biopsies performed in the highest risk patients, who were likely biopsied more frequently than every half year. This study intentionally focused on the utility of long-term RSB in otherwise standard risk patients and did not attempt to address the issue of what should be done in patients deemed to be at higher risk for rejection.

We recognize that patients often undergo biopsy for cellular rejection as part of their regular follow-up cardiac catheterization that also includes screening for AMR, evaluating for cardiac allograft vasculopathy and assessing hemodynamics. We elected to not include AMR in our analysis since much of our data predates standard AMR pathologic classifications. However, we have recently reported our center's recent AMR outcomes which demonstrated an exceedingly low incidence of $\geq$ pAMR 2 biopsies. ${ }^{40}$ Especially for patients in whom long-term routine biopsy is likely low yield, noninvasive methods of surveillance for vasculopathy and graft function deserve further study and consideration. ${ }^{41}$

\section{5 | CONCLUSIONS}

Most pediatric patients do not have significant rejection detected by RSB beyond 2 years after heart transplant. Indiscriminate long-term RSB in pediatric heart transplant should be reexamined given the low rate of detected rejection. Age at transplant may 
FIGURE 3 Kaplan-Meier curves for time to first grade $\geq 3 \mathrm{~A}$ rejection during follow-up beyond 2 y post-transplant by levels of baseline characteristics, truncated at Year 10
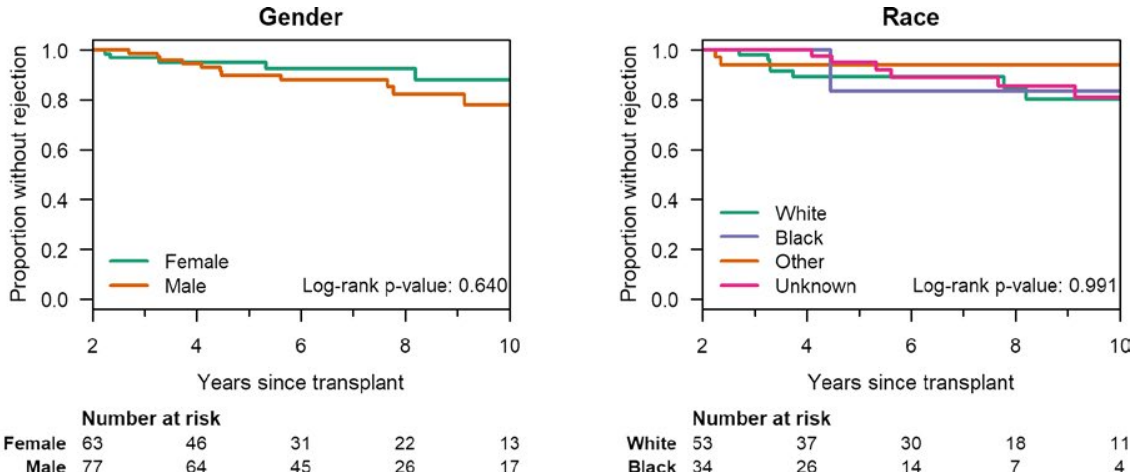
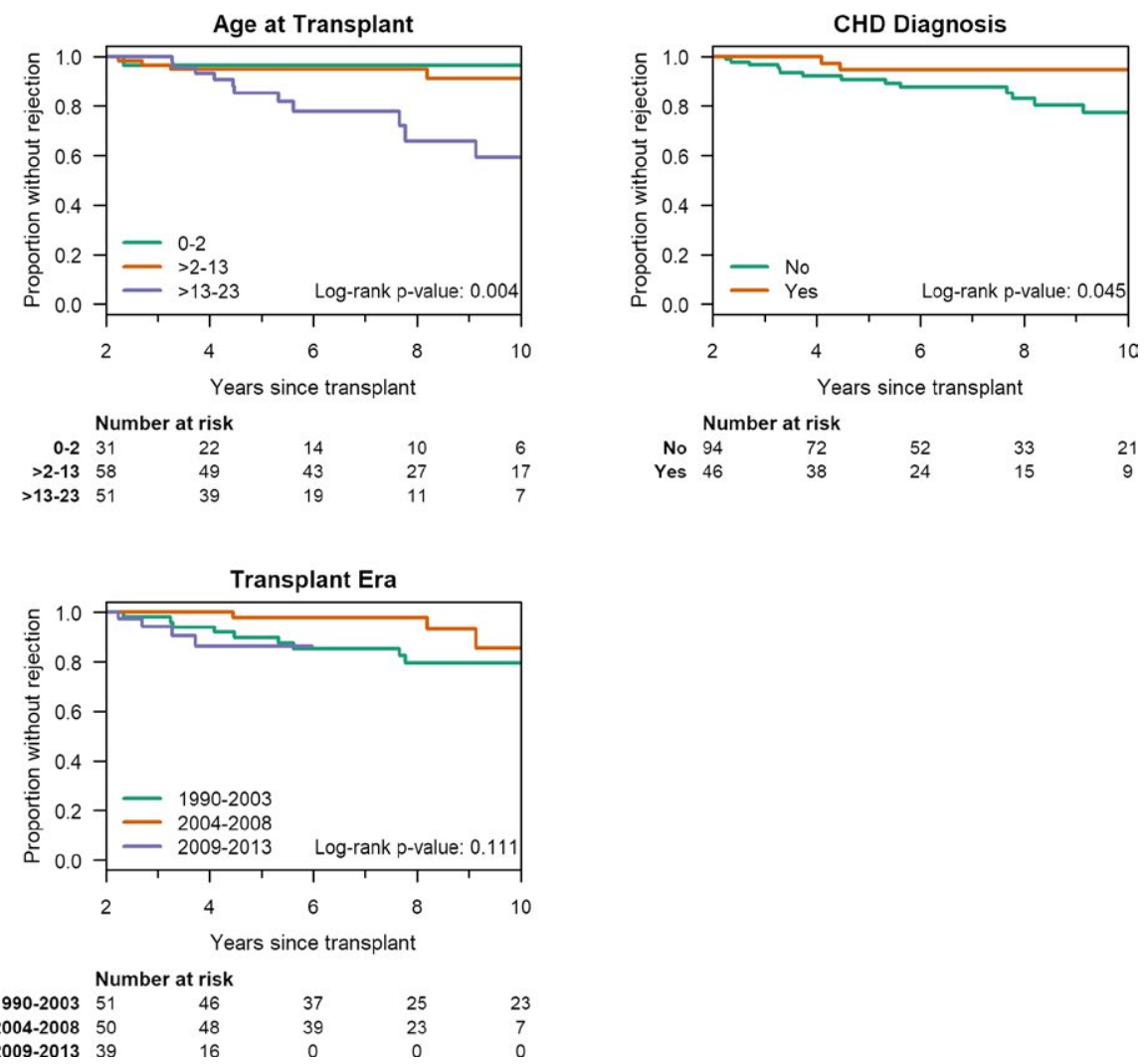

be an important consideration when determining patient-specific long-term surveillance plans. Prospective evaluation of different long-term surveillance strategies may be warranted.

TAB LE 3 Estimated HRs from multivariable Cox proportional hazards model, based on exhaustive search results

\begin{tabular}{|c|c|c|c|c|}
\hline Covariates & HR & $95 \% \mathrm{Cl}$ & $P$-value & Global $P^{*}$ \\
\hline \multicolumn{5}{|c|}{ Age at transplant (y) } \\
\hline $0-2$ & 1.00 & REF & REF & 0.008 \\
\hline$>2-13$ & 2.12 & $(0.25,17.7)$ & 0.490 & \\
\hline$>13-23$ & 7.61 & $(0.99,58.8)$ & 0.052 & \\
\hline CHD diagnosis & 0.26 & $(0.06,1.15)$ & 0.076 & 0.076 \\
\hline
\end{tabular}

"Global P-value obtained from likelihood ratio test.

\section{CONFLICT OF INTEREST}

None of the authors have any relevant disclosures. This project was not funded.

\section{AUTHORS' CONTRIBUTIONS}

David M. Peng, Victoria Y. Ding, Seth A. Hollander, David N. Rosenthal, Christopher S. Almond, Charlotte Sakarovitch, Manisha Desai, and Doff B. McElhinney: Involved in concept/design; David M. Peng, Seth A. Hollander, and Tigran Khalapyan: Collected data; David M. Peng, Victoria Y. Ding, Charlotte Sakarovitch, Manisha Desai, and Doff B. McElhinney: Analyzed and interpreted the data; David M. Peng: Drafted the article; Victoria Y. Ding, Seth A. Hollander, John C. Dykes, David N. Rosenthal, Christopher S. Almond, Charlotte 
Sakarovitch, Manisha Desai, and Doff B. McElhinney: Critically revised the article; Victoria Y. Ding, Seth A. Hollander, John C. Dykes, David N. Rosenthal, Christopher S. Almond, Charlotte Sakarovitch, Manisha Desai, and Doff B. McElhinney: Approved the article.

\section{ORCID}

David M. Peng (iD https://orcid.org/0000-0001-7763-7518

\section{REFERENCES}

1. Caves PK, Stinson EB, Graham AF, Billingham ME, Grehl TM, Shumway NE. Percutaneous transvenous endomyocardial biopsy. JAMA. 1973;225:288-291.

2. Patel JK, Kobashigawa JA. Should we be doing routine biopsy after heart transplantation in a new era of anti-rejection? Curr Opin Cardiol. 2006;21:127-131.

3. Lampert BC, Teuteberg JJ, Shullo MA, Holtz J, Smith KJ. Cost-effectiveness of routine surveillance endomyocardial biopsy after 12 months post-heart transplantation. Circ Heart Fail. 2014;7:807-813.

4. Fiorelli Al, Benvenuti L, Aielo V, et al. Comparative analysis of the complications of 5347 endomyocardial biopsies applied to patients after heart transplantation and with cardiomyopathies: a singlecenter study. Transplant Proc. 2012;44:2473-2478.

5. Mital S. Value of surveillance biopsy in pediatric heart transplantation. Pediatr Transplant. 2007;11:233-235.

6. Kirklin JK, Naftel DC, Bourge RC, et al. Rejection after cardiac transplantation. A time-related risk factor analysis. Circulation. 1992;86:li236-41.

7. Wagner K, Oliver MC, Boyle GJ, et al. Endomyocardial biopsy in pediatric heart transplant recipients: a useful exercise? (Analysis of 1,169 biopsies). Pediatr Transplant. 2000;4:186-192.

8. Pahl E, Naftel DC, Canter CE, Frazier EA, Kirklin JK, Morrow WR. Death after rejection with severe hemodynamic compromise in pediatric heart transplant recipients: a multi-institutional study. J Heart Lung Transplant. 2001;20:279-287.

9. Shah KB, Flattery MP, Smallfield MC, et al. Surveillance endomyocardial biopsy in the modern era produces low diagnostic yield for cardiac allograft rejection. Transplantation. 2015;99:e75-e80.

10. Chi $\mathrm{NH}$, Chou NK, Tsao $\mathrm{Cl}$, et al. Endomyocardial biopsy in heart transplantation: schedule or event? Transplant Proc. 2012;44:894-896.

11. Söderlund C, Rådegran G. Acute cellular rejection later than one year after heart transplantation: a single-center retrospective study at Skåne University Hospital in Lund 1988-2010. Clin Transplant. 2017;31:e12998.

12. Orrego CM, Cordero-Reyes AM, Estep JD, Loebe M, Torre-Amione $G$. Usefulness of routine surveillance endomyocardial biopsy 6 months after heart transplantation. J Heart Lung Transplant. 2012;31:845-849.

13. Stehlik J, Starling RC, Movsesian MA, et al. Utility of long-term surveillance endomyocardial biopsy: a multi-institutional analysis. J Heart Lung Transplant. 2006;25:1402-1409.

14. Klingenberg R, Koch A, Schnabel PA, et al. Allograft rejection of ISHLT grade $>/=3 \mathrm{~A}$ occurring late after heart transplantation-a distinct entity? J Heart Lung Transplant. 2003;22:1005-1013.

15. Heimansohn DA, Robison RJ, Paris JM III, Matheny RG, Bogdon J, Shaar CJ. Routine surveillance endomyocardial biopsy: late rejection after heart transplantation. Ann Thorac Surg. 1997;64:1231-1236.

16. Brunner-La Rocca HP, Kiowski W. Identification of patients not requiring endomyocardial biopsies late after cardiac transplantation. Transplantation. 1998;65:533-538.
17. Gradek WQ, D'Amico C, Smith AL, Vega D, Book WM. Routine surveillance endomyocardial biopsy continues to detect significant rejection late after heart transplantation. $J$ Heart Lung Transplant. 2001;20:497-502.

18. Kubo SH, Naftel DC, Mills RM, et al. Risk factors for late recurrent rejection after heart transplantation: a multiinstitutional, multivariable analysis. Cardiac Transplant Research Database Group. J Heart Lung Transplant. 1995;14:409-418.

19. Hausen B, Rohde R, Demertzis S, Albes JM, Wahlers T, Schafers HJ. Strategies for routine biopsies in heart transplantation based on 8-year results with more than 13,000 biopsies. Eur J Cardiothorac Surg. 1995;9:592-598.

20. White JA, Guiraudon C, Pflugfelder PW, Kostuk WJ. Routine surveillance myocardial biopsies are unnecessary beyond one year after heart transplantation. J Heart Lung Transplant. 1995;14:1052-1056.

21. Sethi GK, Kosaraju S, Arabia FA, Roasdo LJ, McCarthy MS, Copeland JG. Is it necessary to perform surveillance endomyocardial biopsies in heart transplant recipients? J Heart Lung Transplant. 1995;14:1047-1051.

22. Spratt P, Sivathasan C, Macdonald P, Keogh A, Chang V. Role of routine endomyocardial biopsy to monitor late rejection after heart transplantation. J Heart Lung Transplant. 1991;10:912-914.

23. Winters GL, Costanzo-Nordin MR, O'Sullivan EJ, et al. Predictors of late acute orthotopic heart transplant rejection. Circulation. 1989;80:lii106-10.

24. Webber SA, Naftel DC, Parker J, et al. Late rejection episodes more than 1 year after pediatric heart transplantation: risk factors and outcomes. J Heart Lung Transplant. 2003;22:869-875.

25. Chin C, Naftel DC, Singh TP, et al. Risk factors for recurrent rejection in pediatric heart transplantation: a multicenter experience. $J$ Heart Lung Transplant. 2004;23:178-185.

26. Zinn MD, Wallendorf MJ, Simpson KE, Osborne AD, Kirklin JK, Canter CE. Impact of age on incidence and prevalence of moderate-to-severe cellular rejection detected by routine surveillance biopsy in pediatric heart transplantation. J Heart Lung Transplant. 2017;36:451-456

27. Kuhn MA, Deming DD, Cephus CE, et al. Moderate acute rejection detected during annual catheterization in pediatric heart transplant recipients. J Heart Lung Transplant. 2003;22:276-280.

28. Rosenthal DN, Chin C, Nishimura K, et al. Identifying cardiac transplant rejection in children: diagnostic utility of echocardiography, right heart catheterization and endomyocardial biopsy data. J Heart Lung Transplant. 2004;23:323-329.

29. Leonard HC, O'Sullivan JJ, Dark JH. Long-term follow-up of pediatric cardiac transplant recipients on a steroid-free regime: the role of endomyocardial biopsy. J Heart Lung Transplant. 2000;19:469-472.

30. Chin C, Akhtar MJ, Rosenthal DN, Bernstein D. Safety and utility of the routine surveillance biopsy in pediatric patients 2 years after heart transplantation. J Pediatr. 2000;136:238-242.

31. Kertesz NJ, Denfield SW, Kearney DL, Towbin JA, Price JK, Gajarski RJ. Usefulness of routine surveillance biopsies in children more than one year after orthotopic heart transplantation. Am J Cardiol. 2001;87:667-668, a11.

32. Balzer DT, Moorhead S, Saffitz JE, Huddleston CB, Spray TL, Canter CE. Utility of surveillance biopsies in infant heart transplant recipients. J Heart Lung Transplant. 1995;14:1095-1101.

33. Zales VR, Crawford S, Backer CL, et al. Role of endomyocardial biopsy in rejection surveillance after heart transplantation in neonates and children. J Am Coll Cardiol. 1994;23:766-771.

34. Braunlin EA, Shumway SJ, Bolman RM, et al. Usefulness of surveillance endomyocardial biopsy after pediatric cardiac transplantation. Clin Transplant. 1998;12:184-189.

35. Levi DS, DeConde AS, Fishbein MC, Burch C, Alejos JC, Wetzel GT. The yield of surveillance endomyocardial biopsies as a screen 
for cellular rejection in pediatric heart transplant patients. Pediatr Transplant. 2004;8:22-28.

36. Reinhartz O, Maeda K, Reitz BA, et al. Changes in risk profile over time in the population of a pediatric heart transplant program. Ann Thorac Surg. 2015;100:989-994; discussion 995.

37. Billingham ME, Cary NR, Hammond ME, et al. A working formulation for the standardization of nomenclature in the diagnosis of heart and lung rejection: Heart Rejection Study Group. The International Society for Heart Transplantation. J Heart Transplant. 1990;9:587-593.

38. Stewart S, Winters GL, Fishbein MC, et al. Revision of the 1990 working formulation for the standardization of nomenclature in the diagnosis of heart rejection. J Heart Lung Transplant. 2005;24:1710-1720.

39. Rossano JW, Dipchand Al, Edwards LB, et al. The Registry of the International Society for Heart and Lung Transplantation: Nineteenth Pediatric Heart Transplantation Report-2016; Focus
Theme: Primary Diagnostic Indications for Transplant. J Heart Lung Transplant. 2016;35:1185-1195.

40. Hollander SA, Peng DM, Mills M, et al. Pathological antibody-mediated rejection in pediatric heart transplant recipients: Immunologic risk factors, hemodynamic significance, and outcomes. Pediatr Transplant. 2018;22: e13197.

41. Chih S, Chong AY, Mielniczuk LM, Bhatt DL, Beanlands RS. Allograft vasculopathy: the Achilles' Heel of heart transplantation. J Am Coll Cardiol. 2016;68:80-91.

How to cite this article: Peng DM, Ding VY, Hollander SA, et al. Long-term surveillance biopsy: Is it necessary after pediatric heart transplant?. Pediatr Transplantation.

2019;23:e13330. https://doi.org/10.1111/petr.13330 